\title{
Primary cardiac lymphoma with unusual presentation: A report of two cases
}

\author{
CHIU-FAN CHEN ${ }^{1}$, PIN-PEN HSIEH ${ }^{2}$ and SHYH-JER LIN ${ }^{3}$ \\ Departments of ${ }^{1}$ Chest Medicine, ${ }^{2}$ Pathology and Laboratory Medicine and ${ }^{3}$ Hematology and Oncology, \\ Kaohsiung Veterans General Hospital, Kaohsiung 813, Taiwan, R.O.C.
}

Received November 12, 2016; Accepted December 6, 2016

DOI: $10.3892 / \mathrm{mco} .2017 .1131$

\begin{abstract}
Primary cardiac tumors are rare, with an incidence of $0.056 \%$ according to autopsy reports. The most common type is myxoma, while other types, including sarcoma, lipoma, papillary fibroelastoma, rhabdomyoma, fibroma, hemangioma, teratoma, lymphoma and mesothelioma also occur. Primary cardiac tumors usually cause embolization, pericardial effusion and arrhythmia, leading to heart failure. Only $10 \%$ of primary cardiac tumors are malignant, approximately $95 \%$ of which are sarcomas, while the remaining 5\% are cardiac lymphomas and mesotheliomas. The present study reported a case of primary cardiac lymphoma (PCL) with bilateral renal involvement and a case of PCL with bilateral adrenal gland involvement. The prognosis of PCL is poor due to the low rate of early detection and treatment. The definitive diagnosis is dependent on pathology, and timely treatment with chemotherapy can be effective. The two cases developed life-threatening arrhythmia and responded to the initial chemotherapy. In the first case, complete remission was achieved after finalization of therapy. However, the second case refused further chemotherapy and succumbed to his condition after two months.
\end{abstract}

\section{Introduction}

Cardiac tumors are among the least investigated tumor types in oncology. Primary cardiac tumors are rare with an incidence of $0.056 \%$ according to autopsy reports (1). Approximately $90 \%$ of primary cardiac tumors are benign, the most common type of which is myxoma, while other types, including lipoma, papillary fibroelastoma, rhabdomyoma, fibroma, hemangioma, teratoma, sarcoma, lymphoma and pericardial mesothelioma also occur. The remaining $10 \%$ of primary cardiac tumors are

Correspondence to: Dr Shyh-Jer Lin, Department of Hematology and Oncology, Kaohsiung Veterans General Hospital, 386 Ta Chung 1st Road, Kaohsiung 813, Taiwan, R.O.C.

E-mail: sjlin@vghks.gov.tw

Key words: heart neoplasm, non-Hodgkin lymphoma, primary cardiac lymphoma malignant, $95 \%$ of which are sarcomas and $5 \%$ of which are cardiac lymphomas and mesotheliomas (2).

Primary cardiac lymphoma (PCL) is defined as non-Hodgkin lymphoma (NHL) involving only the heart and/or pericardium (strict criteria), or as NHL presenting with cardiac manifestations, particularly when the bulk of the tumor is found in the heart (loose criteria) (3). It is estimated that PCLs account for $1.3 \%$ of primary cardiac tumors and $0.5 \%$ of all extranodal NHLs (4). During 1949-2009, only 197 cases of PCL were reported in the literature. By contrast, secondary cardiac involvement of lymphomas is more common, with an incidence of $9-24 \%$ in disseminated and terminal-stage NHL cases (3).

\section{Case reports}

Case 1. A 46-year-old Taiwanese male patient presented with exertional dyspnea for 1 week. The patient denied any history of systemic disease and physical examination showed no remarkable abnormalities. Laboratory examination showed increased levels of creatinine $(2.5 \mathrm{mg} / \mathrm{dl}$; normal range, $0.7-1.5 \mathrm{mg} / \mathrm{dl}$ for male and $0.5-1.2 \mathrm{mg} / \mathrm{dl}$ for female), lactate dehydrogenase (LDH $603 \mathrm{U} / 1$; normal range, 95-213 U/1), aspartate aminotransferase (164 U/1; normal range, 5-35 U/1) and alanine aminotransferase (218 U/1; normal range, 0-40 U/1). Chest X-ray (CXR) showed cardiomegaly with mild left pleural effusion. Electrocardiography (ECG) showed Mobitz type-I second-degree atrioventricular (AV) block. Transthoracic echocardiography (TTE) revealed a left ventricular ejection fraction (LVEF) of $55 \%$, and a right atrial tumor with pericardial and retrocardiac involvement. Computed tomography (CT) also revealed infiltrative tumors involving the right atrium (RA), pericardium, para-aortic region and bilateral kidneys (Fig. 1). Sudden collapse with ventricular fibrillation occurred during hospitalization, and cardiopulmonary resuscitation with defibrillation was performed. After restoration of circulation, subsequent ECG showed a 2:1 AV block, and a temporary pacemaker was inserted. Intermittent ventricular tachycardia (VT) was still noted and was treated with amiodarone together with synchronized cardioversion. Transesophageal echocardiogram (TEE) showed a cardiac tumor involving the anterior mediastinum, aortic root and pulmonary artery root with extension into the RA. Right coronary artery (RCA) encompression was also noted. Thoracoscopic pericardial biopsy showed diffuse large B-cell lymphoma (Fig. 2). The 


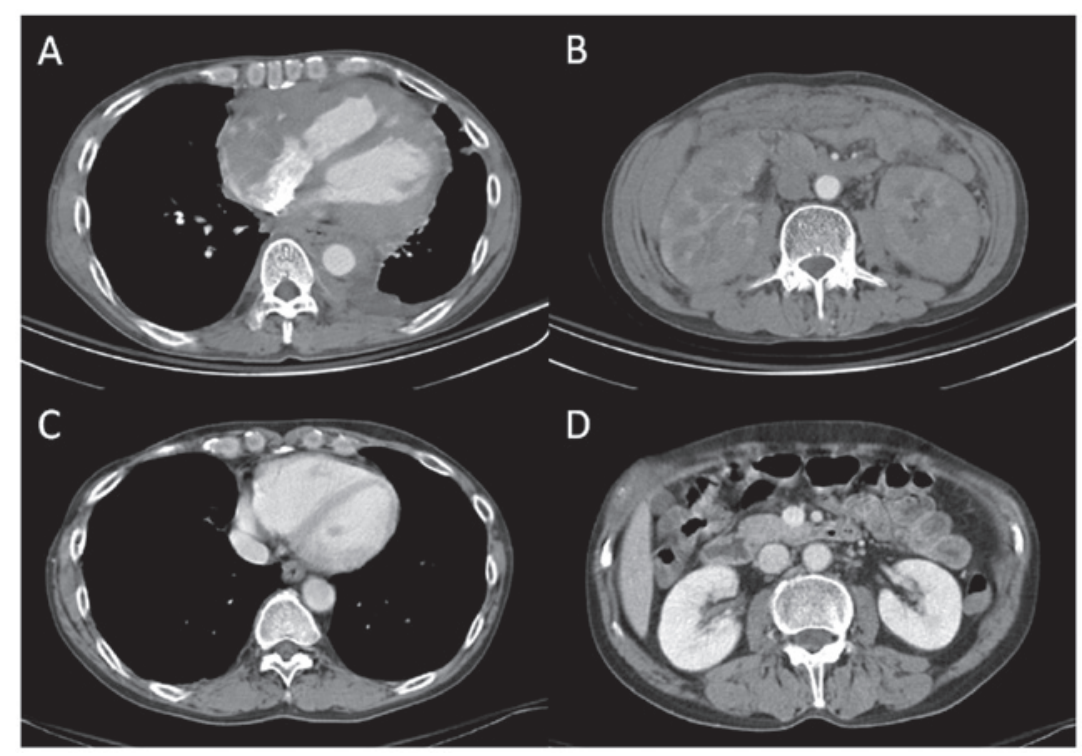

Figure 1. CT scans revealing infiltrative tumors involving (A) the right atrium, pericardium and para-aortic region, and (B) bilateral kidneys. CT scans after seven courses of chemotherapy showed complete remission of lymphoma in (C) the heart and (D) kidneys. CT, computed tomography.

specimen comprised diffuse infiltration of large atypical lymphocytes with centroblastic features. Neoplastic cells were reactive for the B-cell markers CD20 and paired box protein 5 as well as leukocyte common antigen (LCA, CD45), while cells were negative for T-cell marker CD3. Bone marrow examination was negative for lymphoma. Chemotherapy with cyclophosphamide/epirubicin/oncovin/prednisone (CEOP) was initiated, to which the cardiac and renal lymphoma showed a rapid response. The patient's ECG returned to a normal sinus rhythm and renal function was also normalized. The final diagnosis was primary cardiac lymphoma, diffuse large B-cell type, with bilateral kidney involvement, Ann Arbor stage IVa, and IPI risk score 3 (5). During the following 6 months, the patient received 1 cycle chemotherapy with intrathecal methotrexate, and rituximab plus 7 cycles of CEOP (R-CEOP). Re-evaluation by CT scan showed complete remission of lymphoma in heart and kidneys (Fig. 1). The patient remained free of lymphoma for 3 years. Until recently, left hemiparesis due to brain metastasis with lymphoma was found. CT exam of abdomen and chest showed no local recurrence of lymphoma. The patient underwent high-dose methotrexate and rituximab therapy $\mathrm{x} 3$ and with almost complete remission.

Case 2. A 58-year-old Taiwanese male patient presented with intermittent dizziness and syncope up to 10 times with each event lasting 10-15 sec. The patient also had low-grade fever, sore throat, rhinorrhea and general malaise persisting for 2 weeks. He then developed sustained VT, which improved after amiodarone treatment. A grade-III/VI diastolic murmur at the left lower sternal border was noted. Laboratory test revealed increased levels of LDH (449 U/l) and CXR revealed marked cardiomegaly. TTE showed an LVEF of 52\%, moderate to severe AR and a small amount of pericardial effusion. Chest and heart CT revealed tumors involving the RA, right ventricle and bilateral adrenal glands with hemopericardium (Fig. 3). TEE further revealed an epicardial mass around the lower RA, near the AV groove with right ventricular extension and RCA

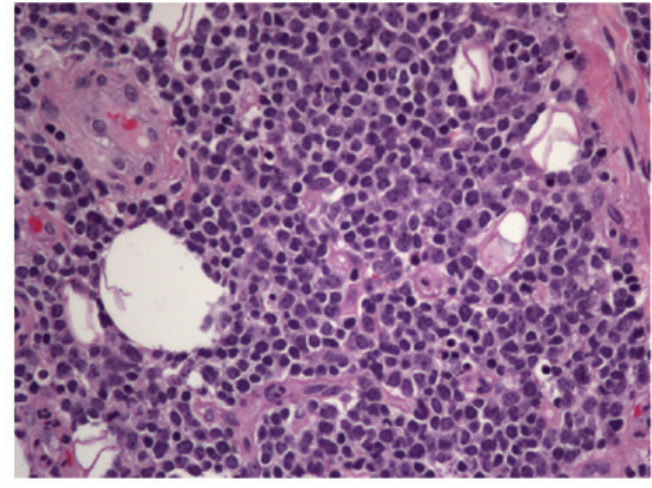

Figure 2. Histological examination revealed diffuse infiltration of large atypical lymphocytes in the pericardial tissue with round or irregular nuclei. Some of the lymphocytes had centroblastic features (hematoxylin and eosin staining; magnification, $\mathrm{x} 400$ ).

encasement. Adrenal gland biopsy confirmed diffuse large B-cell lymphoma (Fig. 4). Immunohistochemistry revealed positivity for LCA and CD20 but negativity for CD3 and cytokeratin AE1/AE3. Bone marrow analysis showed negativity for lymphoma. On the thirteenth day of admission, another episode of sustained VT occurred and was controlled by amiodarone pump therapy. Due to confirmed diagnosis of PCL, diffuse large B-cell type, with bilateral adrenal gland involvement, Ann Arbor stage IVb, and IPI risk score 3, the patient received 1 cycle of first-line chemotherapy with R-CEOP. The patient refused further lymphoma treatment. Follow-up heart CT showed partial regression of cardiac and adrenal lymphoma (Fig. 3). However, despite the good initial response to chemotherapy, the patient refused further lymphoma treatment. He succumbed to VT at 2 months after diagnosis.

\section{Discussion}

The major clinical features of PCL include dyspnea, heart failure, precordial pain, life-threatening arrhythmia due 


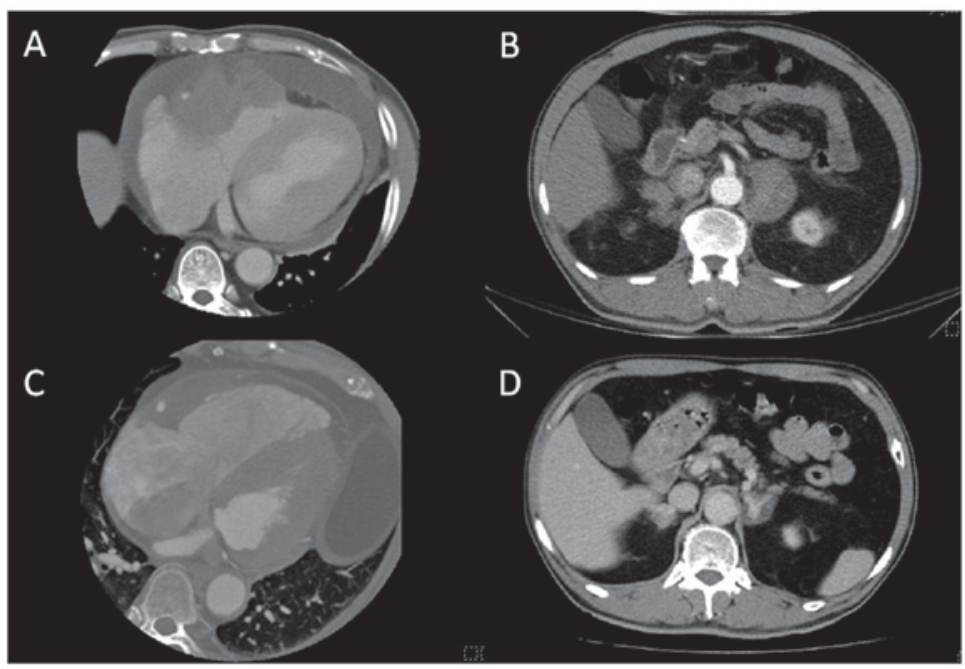

Figure 3. Heart CT showing a diffuse infiltrative and enhanced lesion, mainly from the atrioventricular groove with focal thickening of the right ventricular and right atrial myocardium. (A) The right ventricular outflow tract was also involved and hemopericardium was present. (B) Chest CT also showed bilateral adrenal gland tumors. (C and D) CT scans after first chemotherapy showed partial regression of lymphoma in the heart and adrenal glands. CT, computed tomography.
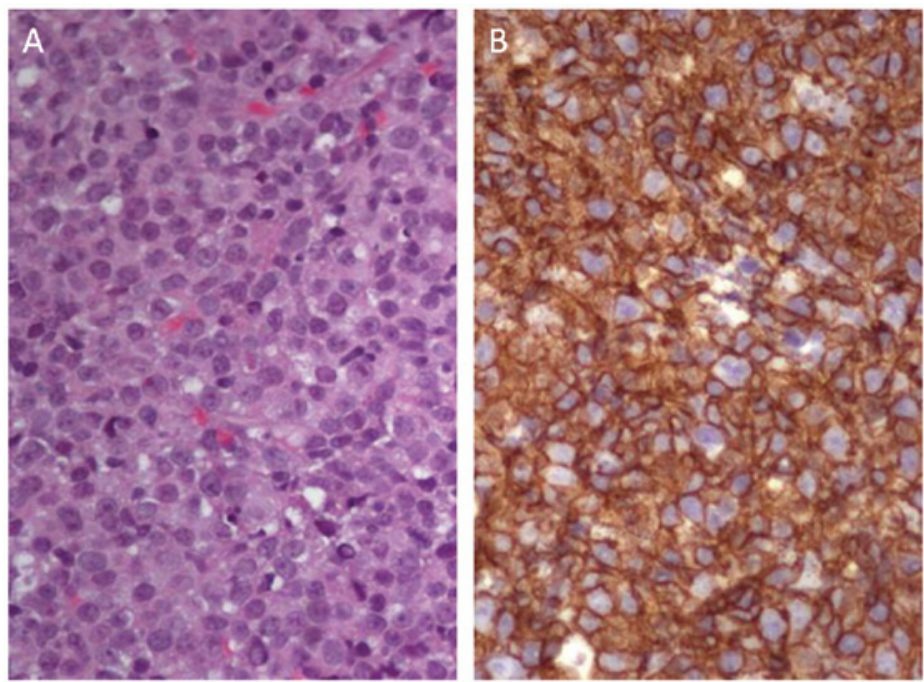

Figure 4. Tissue of adrenal gland biopsy, which confirmed diffuse large B-cell lymphoma. (A) Histological analysis shows large atypical lymphoid cells with round to irregular nuclei, and centroblastic and immunoblastic features (hematoxylin and eosin staining; magnification, x400). (B) Immunohistochemical analysis revealed that the large atypical lymphocytes were positive for CD20 (magnification, x400).

to tumor invasion of the conduction system or irritation of myocardium, pleural effusion, pericardial effusion and shock due to cardiac tamponade or obstruction of blood flow (6). Constitutional symptoms, including fever, chills, sweats and weight loss, are also common. In some PCL cases, patients may develop pulmonary or cerebral embolism. The most common ECG abnormalities include atrial arrhythmias and AV block, with up to $61 \%$ of patients experiencing complete AV block. Other ECG features are right bundle branch block, inverted $\mathrm{T}$ waves, low voltage and life-threatening ventricular tachyarrhythmia (3). CXR usually reveals cardiomegaly or pleural effusion. TTE has a sensitivity of 55-60\% for primary cardiac tumors and TEE has a sensitivity of $97-100 \%$. The sensitivity of cardiac magnetic resonance imaging is superior to that of CT (90-92 vs. 71-73\%) (4,7). In 92\% of PCL cases reported, the right heart chambers were involved, predominantly the RA.
By contrast, in only $7 \%$ of cases, the left heart was involved without right heart invasion. In $25 \%$ of PCL cases, the superior vena cava was also affected. Diffuse large B-cell lymphoma is the most common sub-type of PCL (113 cases reported); the remaining sub-types include Burkitt's lymphoma, T-cell lymphoma, small lymphocytic lymphoma and plasmablastic lymphoma (3).

At present, no definite guidelines exist for the management of PCL. Early systemic chemotherapy appears to be the only effective therapy. The major regimen is the same as that for other types of NHL, namely cyclophosphamide/hydroxydaunorubicin/oncovin/prednisone (CHOP) and since 2001, $\mathrm{CHOP}+$ rituximab (3). In the present cases, R-CEOP was used due to concerns regarding the cardiotoxicity of hydroxydaunorubicin. It is difficult to perform a complete surgical resection of PCL, which provides no survival benefit. Chemotherapy 
followed by radiotherapy for PCL may enhance survival, although its efficacy remains to be determined $(4,8)$. The overall response rate of patients with PCL to chemotherapy is $79 \%$ and the complete remission rate is $59 \%$. The median overall survival of patients with PCL is $\sim 12$ months. Poor prognosis is associated with extra-cardiac disease, immunocompromised status, LV involvement and, notably, absence of arrhythmia (3).

In the first case of the present study, the patient initially presented with cardiac and bilateral renal involvement, while the second case presented with cardiac lymphoma with bilateral adrenal gland involvement. It is suggested that these two cases are of PCL with bilateral extra-cardiac organ involvement, as their cardiac tumors involved the right heart and particularly the RA, which is the most common site of PCL. The pathology of diffuse large B-cell lymphoma also represents the most common cell type of PCL. In a review of the recent literature on cardiac lymphoma, only four cases with similar presentations were found. Of these, one was of PCL with bilateral renal involvement in a patient with human immunodeficiency virus infection (9), two were of PCL with left kidney involvement $(9,10)$ and one was of PCL with left adrenal gland involvement (11).

Primary cardiac tumors are rarely encountered by clinical physicians and PCL is even rarer. Although the development of modern imaging technology has improved the detectability of this disease, invasive tissue biopsy and awareness of clinicians of the disease are required for early pathological diagnosis, which is essential for effective treatment. Despite the poor overall survival of affected patients and limited current knowledge regarding specific treatments, early systemic chemotherapy yields a high response rate and improves the possibility of survival. Lymphoma should be considered by clinicians if cardiac tumors are encountered. Although PCL is associated with poor prognosis and life-threatening complications, timely and appropriate treatment can be benefitial.

\section{References}

1. Lam KY, Dickens P and Chan AC: Tumors of the heart. A 20-year experience with a review of 12,485 consecutive autopsies. Arch Pathol Lab Med 117: 1027-1031, 1993.

2. Bruce CJ: Cardiac tumours: Diagnosis and management. Heart 97: 151-160, 2011.

3. Petrich A, Cho SI and Billett H: Primary cardiac lymphoma: An analysis of presentation, treatment and outcome patterns. Cancer 117: 581-589, 2011.

4. Ceresoli GL, Ferreri AJ, Bucci E, Ripa C, Ponzoni M and Villa E: Primary cardiac lymphoma in immunocompetent patients: Diagnostic and therapeutic management. Cancer 80: 1497-1506, 1997.

5. Armitage JO: Staging non-Hodgkin lymphoma. CA Cancer J Clin 55: 368-376, 2005.

6. O'Mahony D, Peikarz RL, Bandettini WP, Arai AE, Wilson WH and Bates SE: Cardiac involvement with lymphoma: A review of the literature. Clin Lymphoma Myeloma 8: 249-252, 2008.

7. Faganello G, Belham M, Thaman R, Blundell J, Eller T and Wilde P: A case of primary cardiac lymphoma: Analysis of the role of echocardiography in early diagnosis. Echocardiography 24 : 889-892, 2007.

8. Urban BA and Fishman EK: Renal Lymphoma: CT patterns with emphasis on helical CT. Radiographics 20: 197-212, 2000.

9. Gardiner DS and Lindop GB: Coronary artery aneurysm due to primary cardiac lymphoma. Histopathology 15: 537-540, 1989.

10. Morillas P, Quiles J, Nuñez D, Senent C, Martínez S, Fernández JA and Bertomeu V: Complete regression of cardiac non-Hodgkin lymphoma. Int J Cardiol 106: 426-427, 2006.

11. Memon AQ and Xavier L: Malignant lymphoma of the heart. Featured echocardiogram and case report. Cardiol Rev 10: 323-325, 2002 . 